\title{
Profils Des Femmes A Consommation Problematique De Drogues En Cote d'Ivoire
}

\author{
Dje Bi Tchan Guillaume, (Dr) \\ Département de Psychologie, \\ Université Félix Houphouët Abidjan Cocody, Cote d'Ivoire \\ Coulibaly Onata Chaka, (MA) \\ Département de Psychologie, \\ Université Félix Houphouët Boigny, Cote d'Ivoire
}

Doi: 10.19044/esj.2017.v13n29p306 URL:http://dx.doi.org/10.19044/esj.2017.v13n29p306

\begin{abstract}
Drug use is growing rapidly in Côte d'Ivoire, leading to serious adaptation difficulties and mental health problems. The present study aims at presenting the profile of women with drug use problem in Côte d'Ivoire. The participants, aged 14 to 67 years, were 83 patients of the Blue Cross of Côte d'Ivoire. Data related to drug use were obtained through urine tests (Rapitest) and semi-directive interviews with patients and their resource persons. The results indicate three profiles of women with problematic use. They also indicate that $4 / 5$ of these women are poly-consumers. Considering these results, and given the growing interest of women in decision-making structures, it seems useful to reinforce measures to prevent and / or care for girls in drug problem.
\end{abstract}

Keywords: Women's profiles, drug, problematic use, poly-consumer

\section{Résumé}

La consommation de drogues connait une forte croissance en Côte d'Ivoire, entrainant d'importantes difficultés d'adaptation et des problèmes de santé mentale. L'objectif de la étude est de présenter le profil des femmes à consommation problématique de drogues en Côte d'Ivoire. Elle a porté sur 83 patientes de la Croix Bleue Côte d'Ivoire, âgées de 14 à 67 ans. Les données relatives à la consommation de drogues ont été obtenues à l'aide des tests d'urine (Rapitest) et des entretiens semi-directifs avec les patientes et leurs personnes ressources. Les résultats indiquent trois profils de femmes à consommation problématique. Ils indiquent également que les 4/5 de ces femmes sont polyconsommatrices. Au regard de ces résultats, et vu l'intérêt de plus en plus important accordé aux femmes dans les instances de décision, 
il nous semble utile de renforcer les mesures de prévention et/ou de prises en charge des jeunes filles dans la problématique des drogues.

Mots-clés: Profils des femmes, drogue, consommation problématique, polyconsommatrice

\section{Introduction}

L'usage de drogues, phénomène au départ convivial et social, est devenu un problème de santé publique, mieux un problème socioéconomique pour les Etats en développement qui, pour la plupart, sont caractérisés par des transformations sociales rapides et des indices de pauvreté importants. Le résumé du rapport mondial sur les drogues, publié par l'ONUDC en 2016, révèle que la consommation de drogues compromet la dimension du développement durable se rapportant à l'égalité des sexes et à l'autonomisation des femmes et des filles. Il existe des différences entre les hommes et les femmes concernant les drogues de prédilection et les vulnérabilités aux drogues. Les femmes toxicomanes et atteintes par le VIH sont plus vulnérables et plus stigmatisées que les hommes. Elles sont plus exposées aux violences et maltraitances de la communauté. Les modèles de rôles sociaux (Education, position sociale, conditions de travail, statut matrimonial, charges domestiques...) étant variables selon le sexe, les communautés sont généralement moins laxistes quant aux sanctions à l'encontre de la femme consommatrice de substances psychoactives (Coppel, 2004). Pour le même type de consommation, comparées aux hommes, les femmes sont souvent considérées comme des déviantes, et donc stigmatisées. De ce fait, elles seraient plus enclines à manifester des troubles de santé mentale. C'est ce que semble également traduire (Stocco, 2007) lorsqu'il mentionne que les femmes qui présentent une consommation problématique de drogues connaissent des difficultés particulières, et nécessitant des interventions adaptées. Il y a dès lors un intérêt que l'on focalise son attention sur la consommation de drogues chez les femmes. La consommation problématique de drogue, c'est lorsqu'une personne continue de prendre des drogues, même si cela lui cause des problèmes de santé et des problèmes psychosociaux tels qu'une mauvaise performance au travail, des relations difficiles avec ses amis, des ennuis financiers ou l'abandon de certaines activités (Landry, Tremblay, Guyon, Bergeron, \& Brunelle, 2004).

La consommation problématique serait plus fragilisante pour les femmes. Or, en l'état actuel de nos connaissances, les statistiques obtenues en Côte d'Ivoire faisant l'état des consommations problématiques des substances psychoactives chez les femmes, et présentant la relation entre ces consommations et les questions de santé mentale, sont presqu'inexistantes. En conséquence, vu l'intérêt de plus en plus important, à elles, accordé dans 
les politiques de développement, il nous semble utile d'identifier dans le présent chapitre les profils des femmes addictes de substances psychoactives en Côte d'Ivoire.

Une telle étude aurait l'avantage de présenter, d'une part la situation de la consommation des substances psychoactives par les femmes en Côte d'Ivoire, d'autre part les caractéristiques de ces femmes. L'on pourrait être ainsi orienté à propos des facteurs à prendre en compte dans la politique de prévention et de prise en charge.

L'objectif de la présente étude est d'identifier les caractéristiques des femmes ayant une consommation problématique de drogues.

\section{Méthodologie}

\section{Participants}

L'étude a été réalisée au Centre d'accueil de la Croix Bleue Côte d'Ivoire. C'est un centre spécialisé pour la prise en charge des personnes ayant des problèmes de consommation de drogues. Les observations ont porté sur l'ensemble des patientes de la période de janvier 2015 à décembre 2016 pour un problème de consommation de drogues. Elles sont au nombre de 83, d'âge compris entre 14 et 67 ans. Elles sont toutes accompagnées par leurs parents pour une demande de prise en charge, au motif que leur consommation perturbe leurs activités quotidiennes.

\section{Instruments}

Les données ont été collectées en nous référant aux dossiers de consultations des patientes, et à l'aide des entretiens à des entretiens semidirectifs.

Le dossier de consultation médicale de chaque patiente est rempli par les infirmiers et complété par le Médecin traitant. Il comporte la fiche de consultation et le résultat au Rapitest. La fiche de consultation donne des informations sur les variables personnelles et situationnelles. Il s'agit précisément des informations relatives à l'âge, au sexe, à la profession, au niveau d'étude, au statut matrimonial, à l'histoire de la consommation et à l'effet recherché par le patient. Le Rapitest Multidrug Pipette Panel, est un test rapide qui détecte la présence simultanée de plusieurs drogues et métabolites de drogue dans l'urine du patient. Son paramétrage est tel qu'il détecte les drogues suivantes : amphétamine, barbituriques, benzodiazépines, buprénorphine, cocaine, cotinine, kétamine, cannabis, méthadone, méthamphétamine, morphine et opiacé, tramadol, antidépresseurs tricycliques.

L'entretien semi-directif porte sur l'histoire de la consommation de chaque femme, sa motivation de consommation, ses mécanismes d'adaptation individuelle et relationnelle. 


\section{Procédure de collecte des données}

Une fois reçues au centre, elles sont soumises à une même procédure de passation de ces instruments. Elles sont reçues par le service social qui donne des informations nécessaires à la prise en charge des patients. Admises à la consultation, elles sont référées aux infirmiers qui renseignent les dossiers de consultation. Le dossier est complété par le Médecin traitant. Celui-ci, à l'issue de cette première consultation, demande un test urinaire, dont le Rapitest, pour la suite des investigations. C'est après cette étape qu'elles soumises aux entretiens semi-directifs.

Les entretiens se déroulent en deux étapes, la première entre une patiente et un psychiatre ou une patiente, un médecin et un psychologue, la seconde entre une patiente, ses personnes ressources et un psychiatre ou une patiente, ses personnes ressources, un médecin et un psychologue. La seconde étape est une consolidation ou un approfondissement des informations obtenues de la première.

Les données issues de cette procédure de recueil ont été traitées à l'aide du logiciel Excel. Le paragraphe suivant est consacré à la présentation des résultats qui en découlent.

\section{Résultats}

Age et consommation problématique de drogues

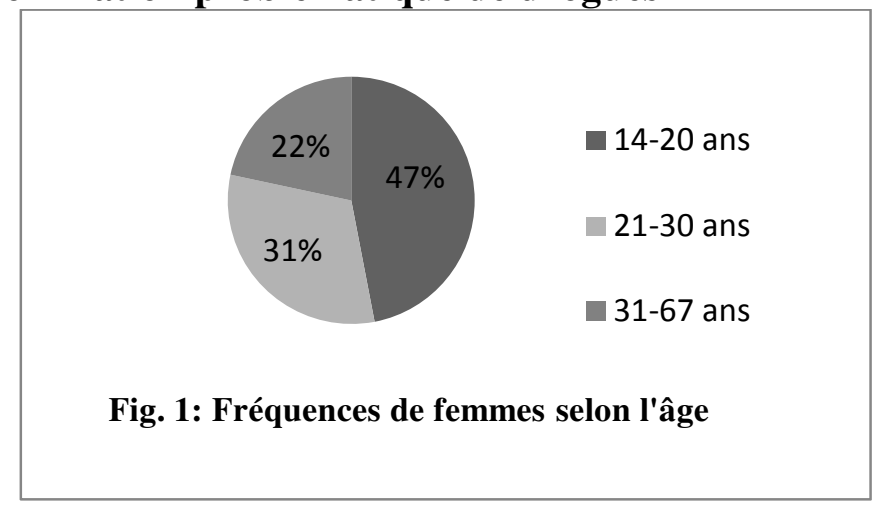

On peut observer que la fréquence de femmes à consommation problématique varie selon l'âge. Elle diminue avec l'âge. Les adolescentes de 14-20 ans sont les consommatrices les plus nombreuses, suivies des femmes de 21-30 ans et de 31-67 ans. En outre, il apparait que plus des 3/4 des consommatrices ont un âge inférieur ou égal à 30 ans. 


\section{Niveau de scolarisation et consommation problématique}

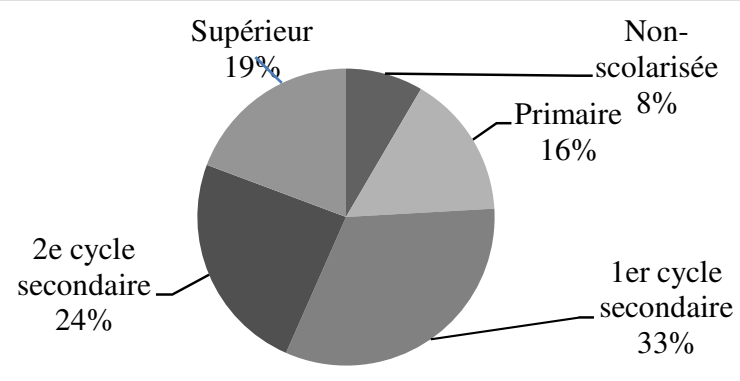

Fig. 2: Fréquences de femmes selon le niveau de scolarisation

La fréquence de femmes augmente avec le niveau de scolarisation, des non-scolarisées aux $1^{\text {er }}$ cycle secondaire, où elle atteint un pic. Ensuite elle connait une diminution jusqu'au niveau supérieur. On relève la fréquence la plus élevée au niveau du $1^{\mathrm{er}}$ cycle secondaire. Ici également, on observe que plus des $3 / 4$ des femmes à consommation problématique ont un niveau d'étude supérieur ou égale au $1^{\mathrm{er}}$ cycle secondaire.

\section{Statut matrimonial et consommation problématique}

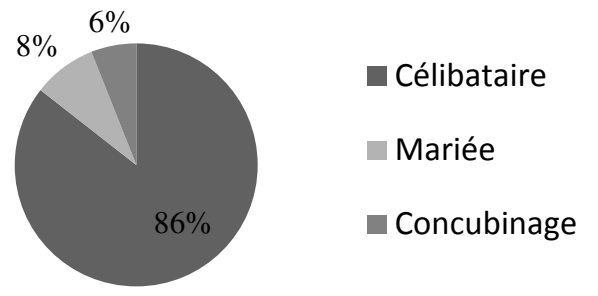

Fig.3: Fréquences de femmes selon le statut matrimonial

La figure 3 montre que les célibataires ont les fréquences les plus élevées en matière de consommation problématique. Elles sont suivies des mariées $(8,43 \%)$ et des femmes vivant en concubinage $(6,02 \%)$. Plus de $4 / 5$ des femmes à consommation problématique sont des célibataires. 


\section{Vue synoptique des drogues consommées}

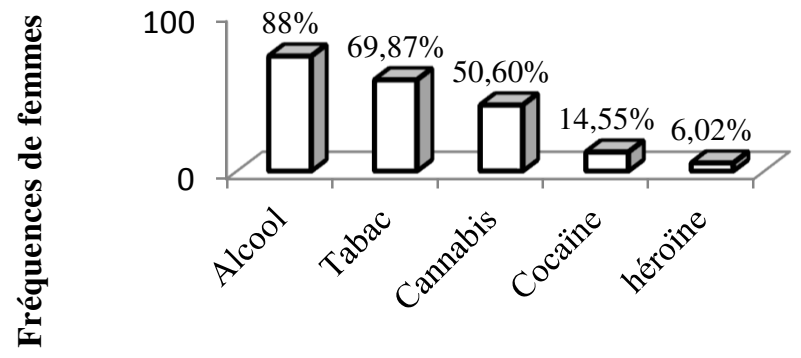

Fig.4: Fréquences de femmes par types de drogues consommées

De la figure 4, on peut retenir que les substances à consommation problématique relevées chez les patientes sont l'alcool, le tabac, le cannabis, la cocaïne et l'héroïne. Les dépendances les plus fréquentes se rapportent à l'alcool (88\%), suivi du tabac (69.87\%) et des drogues illicites, dont le cannabis $(50.6 \%)$. Ces observations nous amènent à penser les patientes examinées ont une polyconsommation de substances psychoactives.

\section{Vue synoptique de la polyconsommation}

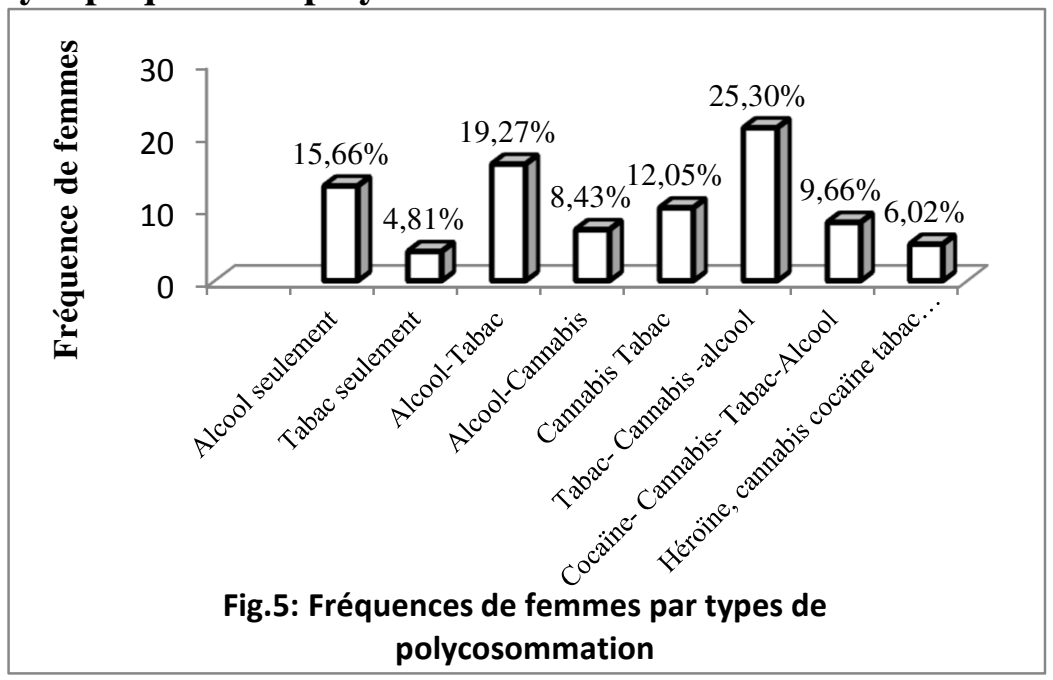

La figure 5 montre que $20,47 \%$ des femmes sont monoconsommatrices (soit $15,66 \%$ pour l'alcool et $4,81 \%$ pour le tabac). Ce qui vient à $79,53 \%$ de polyconsommatrices. En plus, pendant que la dépendance à l'alcool représente la monoconsommation la plus fréquente, l'association tabac-cannabis-alcool représente la polyconsommation la plus 
fréquente. On peut également remarquer que la combinaison CocaïneCannabis-Tabac-Alcool $(9,66 \%)$ et la combinaison Héroïne-CannabisCocaïne-Tabac-Alcool (6,02\%) contiennent chacune au moins deux drogues illicites. Les drogues illicites sont présentes dans les combinaisons de $61,46 \%$ des polyconsommatrices.

\section{Discussion}

La présente étude vise à identifier les profils des femmes à consommation problématique en Côte d'Ivoire. Les analyses ont porté sur les patientes de la Croix bleue Côte d'Ivoire.

Les résultats permettent d'identifier trois profils se rapportant à l'âge, au niveau de scolarisation et au statut matrimonial. Concernant l'âge, on observe plus de femmes à consommation problématique dans l'intervalle 1420 ans. Le phénomène concernerait en majorité les adolescentes. Mieux, plus de $75 \%$ des femmes consommation problématique ont un âge inférieur ou égal à 30 ans. Relativement au niveau de scolarisation, il ressort une fréquence plus élevée chez les femmes de niveau $1^{\text {er }}$ cycle secondaire. Les élèves $\mathrm{du} 1^{\mathrm{er}}$ cycle secondaire de l'enseignement général sont les plus nombreuses à avoir une consommation problématique. Quant au statut matrimonial, la fréquence de célibataires parait la plus élevée.

De ces résultats, en tenant compte de l'âge de scolarisation en Côte d'Ivoire (5-7ans), on peut retenir que ce sont les adolescentes (14-20 ans) des lycées et collèges de l'enseignement général qui sont les plus concernées par la consommation problématique de drogues. La consommation problématique concernerait en majorité les jeunes femmes, avec un début de consommation marqué à l'adolescence, l'âge de la curiosité, de la recherche d'affirmation de soi, mais aussi de l'influence des pairs. Les entretiens semidirectifs avec les participantes révèlent que les lieux et occasions de consommation sont multiples : les quartiers, les fêtes privées, les groupes d'amis de l'école ou les rencontres de jeunes. Les motifs évoqués de manière récurrente sont le désir de se faire accepter par les camarades de classe ou de groupes, ou la peur de se voir marginaliser. L'influence des pairs et le besoin d'endogroupe favoriseraient la consommation de drogues chez ces adolescentes des lycées et collèges. Cette influence est d'autant plus importante que ces adolescentes sont pour la plupart issues de familles recomposées, monoparentales, ou vivent seules. Et les amis constituent pour elles des soutiens permettant de s'affirmer et de modifier leur perception de soi ou de renforcer leur image de soi, qu'elles perçoivent dévalorisée dans leur relation avec leurs familles d'origine. Ces analyses confortent les observations des travaux réalisés dans ce domaine (Barrot, 2008 ; Dubé \& Fournier, 2007 ; Gagnon \& al., 2010). Elles corroborent les analyses faites par les Agences de la santé et des services sociaux de Laval (2012). En effet, 
il apparait de ces travaux qu'à la période adolescente, l'influence des paris contribue, non seulement à l'initiation, mais également à la stabilisation des comportements de consommation de substances psychoactives. A ces observations on peut ajouter que cette influence d'autant plus facile et importante que les sujets se trouvent dans un état de vulnérabilité sociale.

En outre, la fréquence plus importante relevée au niveau $1^{\mathrm{er}}$ cycle secondaire nous fait penser que la drogue ne serait pas consommée du fait de la méconnaissance de ses conséquences. Au contraire, ce sont celles, qui, de par leur instruction, ont des informations à propos des substances psychoactives, auraient tendance à en consommer. C'est dans ce sens que Kuntsche, Rehme \& Gmel (2004) soutiennent que même si l'ivresse reste le plus souvent le fait des femmes les moins éduquées, les femmes les plus éduquées apparaissent les plus concernées par les consommations importantes. Les filles du $1^{\text {er }}$ cycle secondaire observées dans la présente étude sont en majorité en classe de $4^{\text {ème }}$ et de $3^{\text {ème }}\left(3^{\text {ème }}\right.$ et $4^{\text {ème }}$ années du secondaire), et ont des âges compris entre 16 et 18 ans. Elles ont des cursus scolaires irréguliers, avec des retards scolaires et des baisses de performances scolaires. Ce sont ces irrégularités de performances scolaires qui interpellent généralement leurs parents ou leurs personnes ressources, qui les accompagnent au Centre de la Croix Bleue. A l'analyse, il ressort que, ces adolescentes, dans leurs $3^{\text {ème }}$ et $4^{\text {ème }}$ années des lycées et collèges, sont dans une dynamique consolidation de leur appartenance à des groupes d'ami(e)s. Le besoin d'intégration ou de maintien dans ces groupes renforcerait leur propension à expérimenter les pratiques proposées par les membres des groupes. Parmi ces pratiques, se trouve l'initiation à la consommation de substances psychoactives. Et les opportunités de rencontres entre groupes d'ami(e)s étant nombreuses au cours de l'année scolaire, la dépendance s'installe progressivement.

Nous notons également que les consommatrices problématiques sont en majorité des célibataires. Cela s'expliquerait par l'effectif important d'élèves adolescentes, en état de minorité civile, dans l'échantillon examiné. C'est justement leur statut d'élèves qui motive leurs parents ou personnes ressources à les accompagner au centre de la Croix Bleue, lorsque leurs activités scolaires sont perturbées. Si des entretiens avec les élèves célibataires, il ressort les mésadaptations à l'environnement familial et scolaire, avec les femmes célibataires plus âgées, les consommations problématiques semblent liées à l'instabilité émotionnelle et à de mauvaises estimes de soi. Pour ces dernières, la consommation de substances psychoactives serait un remède à la dépression sociale en rapport avec leur profil.

Par ailleurs, nous observons une polyconsommation, qui est d'autant plus préoccupante que les combinaisons comportent des drogues illicites 
telles que le cannabis, la cocaïne et l'héroïne. La consommation de drogues illicites chez les femmes semble une réalité en Côte d'Ivoire. En nous référant à l'âge des participantes de l'étude, il nous parait opportun de suggérer des renforcements des politiques de lutte contre la drogue, notamment dans les établissements scolaires.

Somme toute, les observations faites dans le présent travail nous ont permis d'identifier trois profils de consommatrices problématiques. Le premier profil se rapporte à l'âge. Les consommatrices sont en majorité des adolescentes, d'âges compris entre 14 et 20 ans. Le deuxième profil, le niveau de scolarisation; elles sont de niveau $1^{\text {er }}$ cycle secondaire. Le troisième profil, le statut matrimonial ; elles sont célibataires.

Cependant, notre étude ne nous autorise pas à établir une corrélation entre les profils et la consommation problématique. De tendance descriptive, elle ne nous présente que les caractéristiques des consommatrices. Dans le même sens, nous voulons rappeler que nos observations ont été faites en milieu hospitalier où les informations relatives à la consommation problématique ont été obtenues à l'aide du Rapitest et des entretiens. Même si connaître des perturbations dans ses activités du fait de la consommation de substances psychoactives (Dosage de l'urine à l'aide du Rapitest) constitue un indice important de la consommation problématique chez un sujet, nous pensons que cet indice devrait être consolidé par d'autres observations. En effet, le Rapitest indique la présence ou non de drogues dans l'urine du patient, mais il ne précise pas les fréquences de consommation (Faibles ou Elevées) sur une longue période, 12 mois notamment, comme recommandée par la plus part des tests de dépendance de drogues. Cette précision peut être obtenue si, au cours d'une étude, l'on utilise la grille de dépistage de consommation problématique d'alcool et de drogues chez les adolescents et les adolescentes (DEP-ADO, Version 3.1). Cette grille a l'avantage d'être convenable à une administration collective pour des enquêtes locales ou des études épidémiologiques plus vastes. Une telle étude aura l'avantage d'interroger un échantillon d'une plus grande taille, et ainsi d'identifier d'autres profils de consommateurs. On pourrait définir un objectif explicatif une telle étude, en vue d'identifier des facteurs de dépendance aux drogues chez ces jeunes femmes. Une telle étude se justifierait également dans la mesure où Lambert \& al. (2015) ont obtenu une corrélation entre la consommation problématique des substances psychoactives et les comportements sexuels à risque chez les adolescents.

\section{Conclusion}

Ce travail fait ressortir trois profils de consommatrices problématiques, se rapportant à l'âge, le niveau de scolarisation et le statut matrimonial. Elles sont en majorité des adolescentes, célibataires, et de 
niveau $1^{\text {er }}$ cycle secondaire de l'enseignement général. Elles sont en général dépendantes de l'alcool, du tabac et du cannabis, et présentent une fréquence importante de polyconsommation. Ces profils pourraient constituer un outil d'orientation des politiques de luttes contre la consommation de substances psychoactives.

En outre, Face à ces résultats, en tenant compte du fait que les projections 2020 de la démographie ivoirienne estiment à plus de $37 \%$ les individus dont l'âge varie entre 15 et 35 ans, pour un sex-ratio de 0.98 (Cela signifie qu'il y presqu'autant d'hommes que de femmes dans cette frange de la population), nous proposons que de telles études soient réalisées à une plus grande échelle. Ainsi, l'on disposerait d'informations utiles pour les luttes contre la consommation de drogues chez les jeunes en général, les jeunes femmes en particulier.

\section{References:}

1. Agences de la santé et des services sociaux de Laval (2012). Consommation d'alcool et de drogues des élèves du secondaire. Sélection Santé Laval, vol.9, n 9 .

2. Barrot, J. (2008). La consommation de drogues chez les jeunes vulnérables. Question spécifique, $\mathrm{n}^{\circ} 3$, Observatoire européen des drogues et toxicomanies.

3. Coppel, A. (2004). Figures de femmes. Le courrier des addictions, 6 (2), p. 54-58.

4. Dubé, G. \& Fournier, C. (2007). « Consommation d'alcool et de drogues », In G. Dubé \& col. (2007). Enquête québécoise sur le tabac, l'alcool, la drogue et le jeu chez les élèves du secondaire, 2006, Québec, Institut de la statistique du Québec, chapitre 4, p. 83124.

5. Gagnon, H. \& al. (2010). L'usage de substances psychoactives chez les jeunes Québécois: conséquences et facteurs associés. ISBN : 9782-550-59340-9 (PDf).

6. Kuntsche, E., Rehm, J. \& Gmel, G. (2004). Characteristics of binge drinkers in Europe. Social Science and Medicine, 59 (1), p. 113-127.

7. Lambert, G. \& al. (2015). Consommation problématique de substances psychoactives et comportements sexuels à risque chez les adolescents admis en centre jeunesse. Jeunes et toxicomanies, vol. $14, \mathrm{n}^{\circ} 2$.

8. Landry, M., Tremblay, J., Guyon, L., Bergeron, J. \& Brunelle, N. (2004). La Grille de dépistage de la consommation problématique d'alcool et de drogues chez les adolescents et les adolescentes (DEPADO) : développement et qualités psychométriques. Drogues, santé et société, $\quad$ Vol.3, nº 1, p. 4-86 
9. ONUDC (Office des Nations Unies contre la Drogue et le Crime). (2016). Résumé analytique du rapport mondial sur les drogues.

10. Stocco, P. (2007). Les femmes toxicomanes et la dimension familiale : traitement et questions éthiques. Psychotropes, vol.13, $\mathrm{n}^{\circ} 3$, p. 251-265. 